\title{
Imagerie 3D des lésions kystiques des maxillaires et chirurgie buccale : intérêts de la visualisation isosurfacique
}

\author{
Bornert F, Gros Cl, Dillenseger JP, Féki A, Choquet Ph. \\ (UFR d'Odontologie, Strasbourg)
}

La visualisation mentale d'objets $3 \mathrm{D}$ à partir de descriptions $2 \mathrm{D}$ fait appel à des processus cognitifs complexes (Wu et al., 2012). Cette démarche est essentielle pour entreprendre la chirurgie des kystes des maxillaires. Afin d'explorer la topographie et l'extension des lésions kystiques des maxillaires et préciser certains risques anatomiques, un bilan d'imagerie 3D est fréquemment demandé (Ruhin et al., 2005 ; Sauveur et al., 2008) avec un recours croissant à des appareils de type cone-beam CT. Leur utilisation doit s'inscrire néanmoins dans le cadre de la justification et de l'optimisation de tout examen radiologique (Fortin, 2012).

Plusieurs études montrent que les étudiants de $1^{\text {er }}$ et $2^{\mathrm{e}}$ cycles médicaux n'ont pas tous les mêmes capacités cognitives pour la représentation mentales d'images 3D (Wenzel, 2009) et nécessitent des programmes d'apprentissage adaptés (Sendra-Portero et al., 2012 ; Pascual et al., 2011 ; Vuchkova et al., 2011). Les avantages apportés par l'imagerie $3 \mathrm{D}$ dento-maxillaire et son post-traitement semblent une voie intéressante dans ce domaine.

L'interprétation et l'exploitation des images issues des divers examens de tomographie numérisée 3D se fait alors le plus couramment selon les modalités de visualisation suivante : multiple planar reconstructions (MPR) ou encore par volume rendering (VR) (Boeddinghaus et Whyte, 2008 ; House et al., 2011).

De plus, de nombreuses applications avancées utilisant la représentation d'images $3 \mathrm{D}$ isosurfaciques sont apparues en particulier dans le domaine de la chirurgie maxillo-faciale ainsi qu'en implantologie dentaire associées à des planifications informatiques (CFAO) élaborées (Sannomiya et al., 2008 ; Abboud et al., 2012).

Cependant, peu d'applications concrètes des images isosurfaciques sont décrites à propos des kystes des maxillaires.

Le principe et les limites du post-traitement des images DICOM par l'algorithme de la représentation isosurfacique seront abordés (Lorensen et Cline, 1987).

Plusieurs cas cliniques permettront d'illustrer, au moyen d'images 3D traitées par divers logiciels accessibles (0sirix, Microview $\left.{ }^{\circledR}\right)$, les intérêts chirurgicaux et pédagogiques de cette démarche face à une lésion kystique des maxillaires.

BORNERT Fabien

fabien.bornert@orange.fr

This is an Open Access article distributed under the terms of the Creative Commons Attribution License 2.0, which permits unrestricted use, distribution, and reproduction in any medium, provided the original work is properly cited. 